\title{
Initiating second-line antidiabetic medication among older adults with type 2 diabetes on Metformin
}

\author{
Kristen DeCarlo ${ }^{1}$, Amisha Wallia ${ }^{2,3}$, Raymond H. Kang ${ }^{3}$, Andrew Cooper ${ }^{4}$, Manisha Cherupally ${ }^{3}$, \\ Sterling A. Harris ${ }^{3}$, Cassandra Aikman ${ }^{3}$, David T. Liss ${ }^{3,4}$, Ronald T. Ackermann ${ }^{3,4}$ and Matthew J. O'Brien ${ }^{3,4^{*}}$
}

\begin{abstract}
Background: Antidiabetic medications (ADM), especially sulfonylureas (SFU) and basal insulin (BI), are associated with increased risk of hypoglycemia, which is especially concerning among older adults in poor health. The objective of this study was to investigate prescribing patterns of ADM in older adults according to their health status.

Methods: This case control study analyzed administrative claims between 2013 and 2017 from a large national payer. The study population was derived from a nationwide database of 84,720 U.S. adults aged $\geq 65$, who were enrolled in Medicare Advantage health insurance plans. Participants had type 2 diabetes on metformin monotherapy, and started a second-line ADM during the study period. The exposure was a binary variable for health status, with poor health defined by end-stage medical conditions, dementia, or residence in a long-term nursing facility. The outcome was a variable identifying which second-line ADM class was started, categorized as SFU, BI, or other (i.e. all other ADM classes combined).
\end{abstract}

Results: Over half of participants (54\%) received SFU as initial second-line ADM, 14\% received BI, and 32\% received another ADM. In multivariable models, the odds of filling SFU or BI was higher for participants in poor health than those in good or intermediate health [OR $1.13(95 \% \mathrm{Cl} 1.05-1.21)$ and OR $2.34(95 \% \mathrm{Cl} 2.14-2.55)$, respectively]. SFU and $\mathrm{BI}$ were also more commonly filled by older adults with poor glycemic control.

Conclusions: Despite clinical consensus to use caution prescribing SFU and Bl among older adults in poor health, these medications remain frequently used in this particularly vulnerable population.

Keywords: Type 2 diabetes, Older adults, Antidiabetic medications, Sulfonylureas, Insulin

\section{Background}

The care of older adults with type 2 diabetes presents a growing public health and safety challenge. Diabetes currently affects $27 \%$ of U.S. adults aged 65 years or older, with greater prevalence observed as age increases [1]. Prior research in this population has reported increased

\footnotetext{
*Correspondence: matthew.obrien1@northwestern.edu

${ }^{4}$ Division of General Internal Medicine and Geriatrics, Department

of Medicine, Northwestern University Feinberg School of Medicine, $750 \mathrm{~N}$.

Lake Shore Dr., 10th floor, IL, Chicago, USA

Full list of author information is available at the end of the article
}

risk of diabetes complications, geriatric syndromes, adverse medication events, and associated morbidity and mortality [2]. In light of these concerning data, diabetes care guidelines for older adults recommend developing individualized treatment plans that aim to mitigate these poor outcomes [3-7].

Minimizing hypoglycemia represents a cornerstone of diabetes care in the older population. Older adults are more likely to experience hypoglycemia due to their high rates of polypharmacy, comorbidity, kidney disease, cognitive impairment, and physical frailty, among other risk 
factors $[8,9]$. Hypoglycemia can also result directly from antidiabetic medications (ADM), especially sulfonylureas (SFU) and basal insulin (BI). A recent analysis of nationwide administrative claims data found the highest rates of hypoglycemia associated with these two ADM classes [10]. Despite clinical consensus regarding relaxed glycemic targets among older adults with poor health, several previous studies demonstrate overuse of SFU and BI in this particularly vulnerable population [11-17]. Current diabetes management guidelines address this important area of patient safety by recommending a framework that considers older adults' health status when prescribing these high-risk ADM $[3,4,6,18]$. Specifically, the American Diabetes Association (ADA) and American Geriatrics Society (AGS) consensus report, as well as the recent Endocrine Society Guidelines, suggest higher glycemic targets when using SFU and BI in older adults with poor health, which is defined by the presence of end-stage medical conditions, moderate to severe dementia, functional impairment, or residence in a long-term nursing facility $[3,4,6,18]$.

In this study, we aimed to investigate the magnitude of an existing gap between consensus recommendations about diabetes management in older adults and realworld ADM prescribing patterns in this population. Specifically, we examined fills of SFU, BI, and other ADM classes according to health status in a large national population of adults aged 65 years or older who were enrolled in Medicare Advantage insurance plans.

\section{Methods}

\section{Study Design}

We conducted a case control study using administrative claims data from a large U.S. payer to compare the health status of patients who filled SFU or BI vs. all other ADM alternatives $(\mathrm{N}=84,720)$. To minimize the potential for differences in diabetes duration or severity that may influence ADM prescribing decisions, we restricted our analysis to patients who had only taken metformin before starting one of the follow index medication classes: SFU, BI, dipeptidyl peptidase 4 (DPP-4) inhibitors, glucagonlike peptide-1 (GLP-1) agonists, sodium-glucose cotransporter 2 (SGLT-2) inhibitors, or thiazolidinediones (TZD).

\section{Data and Setting}

The data sources were patients' health plan enrollment files, laboratory claims, pharmacy claims, and medical claims collected by a large health payer between January 1, 2013 and December 31, 2017. Medical diagnoses were coded according to the International Classification of Diseases. Pharmacy claims for individual second-line ADM were grouped into one of the six classes. Hemoglobin
$\mathrm{A}_{1 \mathrm{c}}\left(\mathrm{HbA}_{1 \mathrm{c}}\right)$ values were available for a subset of patients based on their lab vendor. Race/ethnicity is not routinely collected in health plan administrative data sources, but was imputed by the data vendor from regional and other individual characteristics. The data sources studied here were described in-depth previously [19]. We followed the Strengthening the Reporting of Observational Studies in Epidemiology (STROBE) guidelines [20].

\section{Participants}

The study population was derived from a nationwide database of U.S. adults aged $\geq 65$ years, who were enrolled in Medicare Advantage health insurance plans. We included those with: (1) diagnosed type 2 diabetes; (2) a first pharmacy-dispensing event for second-line ADM, defined as the index date; (3) at least 6 months of continuous enrollment before the index date; (4) at least 1 prior fill of metformin during the 6 months before the index date, with no other ADM fills during that time; and (5) at least 1 fill of the index ADM. We considered patients to have type 2 diabetes if they had at least one medical claim associated with a corresponding diagnosis code occurring on or before the index date. We excluded patients with evidence of type 1 diabetes or secondary diabetes prior to the index date, as well as those who filled more than one ADM class on the index date. Detailed definitions of the eligibility criteria, as well as the exposure, are presented in Tables 1 and 2 respectively, with the corresponding diagnosis codes from the International Classification of Diseases [1,21]. Medications comprising the ADM classes studied here are presented in Table 3.

\section{Exposure and Outcomes}

The exposure was a binary variable for health status, adapted from consensus guidelines focused on diabetes management in older adults $[3,4,6]$. Poor health was defined by the presence of at least one medical claim with a diagnosis code for end-stage medical conditions, dementia, or residence in a long-term nursing facility. Patients without evidence of any of these criteria were assumed to be in good or intermediate health. Impairment in activities of daily living could not be assessed using administrative claims data, and was therefore not included in our primary exposure variable. End-stage conditions comprising the guideline definition of poor health was determined by the presence of medical claims associated with diagnosis codes for metastatic cancer, oxygen-dependent lung disease, severe chronic kidney disease (i.e. stages 4, 5, end-stage kidney disease, or dialysis status), and congestive heart failure (Table 3 ) prior to the index date $[3,4,6]$. The outcome was a categorical variable identifying which second-line ADM class was started on the index date, defined by the first fill of the 
Table 1 Definitions for Diabetes-Related Eligibility Criteria

\begin{tabular}{|c|c|c|}
\hline Condition & ICD-9 Codes ${ }^{a}$ & ICD-10 Codes ${ }^{a}$ \\
\hline \multicolumn{3}{|l|}{ Inclusion Criteriab } \\
\hline Type 2 diabetes & $\begin{array}{l}250.00,250.02,250.10,250.12,250.20,250.22,250.30,250.32 \\
\begin{array}{l}250.40,250.42,250.50,250.52,250.60,250.62,250.70,250.72 \\
250.80,250.82,250.90,250.92\end{array}\end{array}$ & $\begin{array}{l}\text { E11.0x, E11.1x, E11.2x, E11.3xxx, E11.4x, E11.5x, E11.6xx, E11.8, } \\
\text { E11.9 }\end{array}$ \\
\hline \multicolumn{3}{|l|}{ Exclusion Criteria } \\
\hline Type 1 diabetes & $\begin{array}{l}250.11,250.13,250.21,250.23,250.31,250.33,250.41,250.43 \\
250.51,250.53,250.61,250.63,250.71,250.73,250.81,250.83 \\
250.91,250.93\end{array}$ & E10.1x, E10.2x, E10.3xxxE10.4x, E10.5x, E10.6xx, E10.8, E10.9 \\
\hline Secondary diabetes & $\begin{array}{l}249.0 x, 249.1 x, 249.2 x, 249.3 x, 249.4 x, 249.5 x, 249.6 x, 249.7 x \\
249.8 x, 249.9 x\end{array}$ & $\begin{array}{l}\text { E08.0x, E08.1x, E08.2x, E08.3xxx, E08.4x, E08.5x, E08.6xx, E08.8, } \\
\text { E08.9 }\end{array}$ \\
\hline $\begin{array}{l}\text { Started }>1 \text { antidiabetic } \\
\text { medication on index } \\
\text { date }^{c}\end{array}$ & $\mathrm{~N} / \mathrm{A}$ & N/A \\
\hline
\end{tabular}

a Diagnosis codes were derived from the International Classification of Diseases, Ninth and Tenth Revisions

b All eligibility criteria, in addition to type 2 diabetes, are described in the manuscript under Participants

${ }^{c}$ Medication fills were assessed using pharmacy claims

Table 2 Definitions for Conditions That Comprise Poor Health Status

\begin{tabular}{|c|c|c|c|}
\hline Condition & ICD-9 Codes & ICD-10 Codes & Billing Codes \\
\hline \multicolumn{4}{|l|}{ End-Stage Medical Conditions } \\
\hline Metastatic cancer & 196.x-199.x & C77.x-C80.x & N/A \\
\hline Oxygen-dependent lung disease & V46.2 & Z99.81 & N/A \\
\hline Chronic kidney disease, stages IV-V & $585.4,585.5$ & N18.4, N18.5 & $\mathrm{N} / \mathrm{A}$ \\
\hline End-stage renal disease & $585.6, \vee 45.11$ & N18.6, Z99.2 & N/A \\
\hline Heart failure $^{a}$ & $\begin{array}{l}\text { 398.91, 402.01, 402.11, 402.91, 404.01, } \\
\text { 404.03, 404.11, 404.13, 404.91, 404.93, } \\
425.4,425.5,425.7,425.8,425.9,428 . x, \\
37.66,37.51,37.52, \text { V42.1, 996.83, } \\
\text { V43.21 }\end{array}$ & 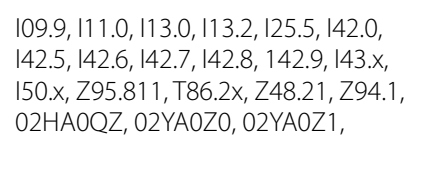 & N/A \\
\hline Dementia & $\begin{array}{l}331.0,331.01,331.11,331.19,331.2 \\
331.7,331.82 \text { 290.0, 290.10, 290.11, } \\
290.12,290.13,290.20,290.21,290.3 \\
290.10,290.41,290.42,290.43,291.2 \\
294.0,294.10,294.11,294.20,294.21 \\
294.8,797\end{array}$ & $\begin{array}{l}\text { F01.50, F01.51, F02.80, F02.81, F03.90, } \\
\text { F03.91, F04, G13.8, F05, F06.1, F06.8, } \\
\text { F10.27, G30.0, G30.1, G30.8, G30.9, } \\
\text { G31.1, G31.2, G31.01, G31.09, G31.83, } \\
\text { G94, R41.81, R54 }\end{array}$ & N/A \\
\hline Long-term nursing facility residence ${ }^{b}$ & & & $\begin{array}{l}\text { 99301-99303, 99311-99313, } \\
\text { 99,315, 99,316, 99,379, 99,380, } \\
\text { G0066 }\end{array}$ \\
\hline
\end{tabular}

${ }^{a}$ Heart failure was defined as any code listed above $+\geq 1$ hospitalization or ED visit for heart failure within 6 months of the index date

${ }^{\mathrm{b}}$ Billing codes for residence in a long-term nursing facility include CPT codes and nursing facility place of service codes

new ADM. Individual index ADM pharmacy claims were assigned to one of the following categories: SFU, BI, or other (i.e. DPP-4, GLP-1, SGLT-2, or TZD).

\section{Covariates}

All covariates were assessed within the 6 months preceding the index date. Demographic data included patients' age, sex, and race/ethnicity. Laboratory values are not routinely available in health plan administrative data sources unless submitted by the laboratory vendor as part of their contract with the health payer. For these data, we were fortunate to have access to a laboratory result for $35 \%$ of submitted laboratory claims nationally. The most recent $\mathrm{HbA}_{1 \mathrm{c}}$ result was categorized: $<8.0 \%, 8.0-10.0 \%$, $>10.0 \%$, and a separate category for those without available values. We used diagnostic codes from inpatient and ambulatory medical claims to examine prevalent microand macrovascular complications prior to the index date. These conditions were grouped according to the Diabetes Complications Severity Index (DCSI) [22], and categorized into the following DCSI scores: $0,1,2$, and $\geq 3$. Cardiovascular codes in the DCSI were excluded because 
Table 3 Individual Medications within Each Antidiabetic Medication Class

\begin{tabular}{|c|c|c|}
\hline Antidiabetic Medication Class & Generic Name & Brand Names \\
\hline \multicolumn{3}{|l|}{ Medications of Interest } \\
\hline \multirow[t]{6}{*}{ SFU } & glipizide & Glucotrol \\
\hline & glyburide & Diabeta, Micronase, Glynase \\
\hline & glicazide & Diamicron \\
\hline & glimepiride & Amaryl \\
\hline & repaglinide & Prandin \\
\hline & nateglinide & Starlix \\
\hline \multirow[t]{4}{*}{ Basal insulin } & insulin glargine & Lantus, Toujeo, Basaglar \\
\hline & insulin detemir & Levemir \\
\hline & insulin degludec & Tresiba \\
\hline & insulin NPH & Humulin, Novolin \\
\hline \multicolumn{3}{|l|}{ Other Medications } \\
\hline \multirow[t]{5}{*}{ DPP-4 } & sitagliptin & Januvia \\
\hline & saxagliptin & Onglyza \\
\hline & alogliptin & Nesina \\
\hline & linagliptin & Tradjenta \\
\hline & vildagliptin & Gliptus, Galvus \\
\hline \multirow[t]{6}{*}{ GLP-1 } & exenatide & Bydureon, Bydureon BCise, Byetta \\
\hline & lixisenatide & Adlyxin \\
\hline & liraglutide & Saxenda, Victoza \\
\hline & albiglutide & Tanzeum \\
\hline & dulaglutide & Trulicity \\
\hline & semaglutide & Ozempic \\
\hline \multirow[t]{3}{*}{ SGLT-2 } & empagliflozin & Jardiance \\
\hline & canagliflozin & Invokana \\
\hline & dapagliflozin & Farxiga \\
\hline \multirow[t]{2}{*}{ TZD } & rosiglitazone & Avandia \\
\hline & pioglitazone & Actos \\
\hline
\end{tabular}

DPP-4 dipeptidyl peptidase-4 inhibitors; GLP-1 glucagon-like peptide 1 receptor agonists; NPH neutral protamine hagedorn; SGLT-2 sodium-glucose cotransporter 2 inhibitors; TZD thiazolidinediones; SFU sulfonylureas and meglitanides

they comprise end-stage conditions that were used to define the primary exposure, thereby reducing the total DCSI score by a maximum of 2 points. Characteristics of prescribers (i.e. family physician, general/internal medicine physician, endocrinologist, nurse practitioner, physician assistant, missing or other) and health coverage (i.e. plan type) were also analyzed as covariates, as well as the year in which the index ADM was prescribed.

\section{Statistical Analysis}

Summary statistics characterized the study population by the class of the index ADM (Table 4), using Chisquare tests to examine associations between baseline covariates and ADM class. Table 5 presents the association of the exposure by index ADM class. The exposure was examined as a dichotomous variable for poor health vs. good or intermediate health, in addition to the individual criteria comprising the definition of poor health (i.e. end-stage comorbidities, dementia, and residence in a long-term nursing facility). Logistic regression was used to model the multivariable association between health status and index ADM class. Two separate multivariable models were constructed, namely: (1) SFU vs. all other ADM alternatives, excluding BI; and (2) BI vs. all other ADM alternatives, excluding SFU.

We conducted a sensitivity analysis excluding the imputed race/ethnicity variable from logistic regression models. We also conducted a sensitivity analysis among only those patients with available $\mathrm{HbA}_{1 \mathrm{c}}$ values prior to the index date. All covariates in this sensitivity analysis were modeled in an identical manner as the primary analysis. All analyses were conducted using [SAS version 9.4, Cary, NC]. The Northwestern University IRB deemed this study to be non-human subjects research, and therefore written informed consent of participants was not required. 
Table 4 Patient Characteristics by Initial Second-Line Antidiabetic Medication Class

\begin{tabular}{|c|c|c|c|c|}
\hline Characteristic $^{\mathrm{a}}$ & $\begin{array}{l}\text { Total } \\
\text { N (\%) }\end{array}$ & $\begin{array}{l}\text { SFU } \\
\text { N (\%) }\end{array}$ & $\begin{array}{l}\mathrm{BI} \\
\mathrm{N}(\%)\end{array}$ & $\begin{array}{l}\text { Other }^{b} \\
\mathrm{~N}(\%)\end{array}$ \\
\hline Total & $84,720(100)$ & $45,734(54)$ & $12,165(14)$ & $26,821(32)$ \\
\hline \multicolumn{5}{|l|}{ Sex } \\
\hline Female & $43,236(51)$ & $22,528(49)$ & $6,683(55)$ & $14,025(52)$ \\
\hline Male & $41,484(49)$ & $23,206(51)$ & $5,482(45)$ & $12,796(48)$ \\
\hline \multicolumn{5}{|l|}{ Age, years } \\
\hline $65-67$ & $22,425(26)$ & $11,540(25)$ & $3,604(30)$ & $7,281(27)$ \\
\hline $68-70$ & $13,145(16)$ & $6,864(15)$ & $1,768(15)$ & $4,513(17)$ \\
\hline $70-74$ & $24,323(29)$ & $13,244(29)$ & $3,219(26)$ & $7,860(29)$ \\
\hline $75-79$ & $13,990(17)$ & $7,843(17)$ & $1,937(16)$ & $4,210(16)$ \\
\hline$\geq 80$ years & $10,837(13)$ & $6,243(14)$ & $1,637(13)$ & 2,957 (11) \\
\hline \multicolumn{5}{|l|}{ Hemoglobin $\mathrm{A}_{1,} \%$} \\
\hline$<8$ & $14,053(17)$ & $7,216(16)$ & $1,081(9)$ & $5,756(21)$ \\
\hline $8-10$ & $11,912(14)$ & $6,507(14)$ & $972(8)$ & $4,433(17)$ \\
\hline$\geq 10$ & $4,079(5)$ & $2,037(4)$ & $802(7)$ & $1,240(5)$ \\
\hline Unavailable $^{c}$ & $54,676(65)$ & $29,974(66)$ & $9,310(77)$ & $15,392(57)$ \\
\hline Mean hemoglobin A1c ( \pm SD), \% ${ }^{d}$ & $7.95(1.62)$ & $7.95(1.57)$ & $8.59(2.14)$ & $7.79(1.48)$ \\
\hline \multicolumn{5}{|l|}{$\begin{array}{l}\text { Diabetes Complications } \\
\text { Severity Index }{ }^{\mathrm{e}} \text { score }\end{array}$} \\
\hline 0 & $47,026(56)$ & $25,946(57)$ & $6,384(52)$ & $14,696(55)$ \\
\hline 1 & $15,783(19)$ & $8,294(18)$ & $2,268(19)$ & $5,221(19)$ \\
\hline 2 & $10,999(13)$ & $5,892(13)$ & $1,615(13)$ & $3,492(13)$ \\
\hline$\geq 3$ & $10,912(13)$ & $5,602(12)$ & $1,898(16)$ & $3,412(13)$ \\
\hline \multicolumn{5}{|l|}{ Prescriber Specialty } \\
\hline Endocrinology & $3,652(4)$ & $1,362(3)$ & $738(6)$ & $1,552(6)$ \\
\hline Family practice & $33,681(40)$ & $18,641(41)$ & $4,595(38)$ & $10,445(39)$ \\
\hline Internal medicine & $29,543(35)$ & $16,467(36)$ & $3,832(32)$ & $9,244(34)$ \\
\hline Nurse/PA & $6,889(8)$ & $3,448(8)$ & $1,141(9)$ & $2,300(9)$ \\
\hline Other/Missing & $10,955(13)$ & $5,816(13)$ & $1,859(15)$ & $3,280(12)$ \\
\hline \multicolumn{5}{|l|}{ Health Plan Type } \\
\hline Health maintenance organization & $25,499(30)$ & $13,585(30)$ & $4,070(33)$ & $7,844(29)$ \\
\hline Preferred provider organization & $8,232(10)$ & $4,612(10)$ & $965(8)$ & $2,655(10)$ \\
\hline Other & $50,989(60)$ & $27,537(60)$ & $7,130(59)$ & $16,322(61)$ \\
\hline \multicolumn{5}{|l|}{ Fill Year } \\
\hline 2013 & $10,112(12)$ & $6,033(13)$ & $1,289(11)$ & $2,790(10)$ \\
\hline 2014 & $11,766(14)$ & 6,585 (14) & $1,653(14)$ & $3,528(13)$ \\
\hline 2015 & $11,046(13)$ & $6,038(13)$ & $1,379(11)$ & $3,629(14)$ \\
\hline 2016 & 27,639 (33) & 14,907 (33) & $4,537(37)$ & $8,195(31)$ \\
\hline 2017 & $24,157(28)$ & $12,171(27)$ & $3,307(27)$ & $8,679(32)$ \\
\hline
\end{tabular}

BI: basal insulin, SFU: sulfonylurea

a All participant characteristics were significantly associated with initial second-line antidiabetic medication class with a P-value of $<0.001$

b The Other category comprises DPP-4 inhibitors, GLP-1 agonists, SGLT-2 inhibitors, and thiazolidinediones (TZDs)

c Laboratory values are not routinely available in health plan administrative data sources unless submitted by the laboratory vendor as part of their contract with the health payer. For these data, $35 \%$ of submitted laboratory claims nationally included a result. The Unavailable category includes patients without evidence of a test (no claim), as well as those with evidence of a test but no available result

d Mean hemoglobin A1c ( \pm SD) was calculated for all participants with available values

e The Diabetes Complications Severity Index (DCSI) is a validated index composed of micro- and macrovascular complications of diabetes. In this analysis, we modified the DCSI by removing cardiovascular diagnoses that comprise the primary exposure variable. Scores were categorized as $0,1,2$, and $\geq 3$ 
Table 5 Poor Health Status and Its Individual Components by Initial Second-Line Antidiabetic Medication Class

\begin{tabular}{|c|c|c|c|c|}
\hline $\begin{array}{l}\text { Health status } \\
\text { variables }^{\mathrm{a}}\end{array}$ & $\begin{array}{l}\text { Total } \\
\mathrm{N}(\%)\end{array}$ & $\begin{array}{l}\text { SFU } \\
N(\%)\end{array}$ & $\begin{array}{l}\text { BI } \\
\mathrm{N}(\%)\end{array}$ & $\begin{array}{l}\text { Other } \\
\mathrm{N}(\%)\end{array}$ \\
\hline \multicolumn{5}{|l|}{ Poor health ${ }^{b}$} \\
\hline No & $79,544(94)$ & $43,199(94)$ & $10,788(89)$ & $25,557(95)$ \\
\hline Yes & $5,176(6)$ & $2,535(6)$ & $1,377(11)$ & $1,264(5)$ \\
\hline \multicolumn{5}{|c|}{ End-stage conditions ${ }^{c}$} \\
\hline No & $82,810(98)$ & $44,712(98)$ & $11,733(96)$ & $26,365(98)$ \\
\hline Yes & $1,910(2)$ & $1,022(2)$ & $432(4)$ & $456(2)$ \\
\hline \multicolumn{5}{|l|}{ Dementia $^{d}$} \\
\hline No & $82,391(97)$ & $44,597(98)$ & $11,579(95)$ & $26,215(98)$ \\
\hline Yes & $2,329(3)$ & $1,137(2)$ & $586(5)$ & $606(2)$ \\
\hline \multicolumn{5}{|c|}{ Residence in nursing facility ${ }^{\mathrm{e}}$} \\
\hline No & $82,631(98)$ & $44,860(98)$ & $11,414(94)$ & $26,357(98)$ \\
\hline Yes & $2,089(2)$ & $874(2)$ & $751(6)$ & $464(2)$ \\
\hline
\end{tabular}

$B /$ basal insulin, SFU sulfonylurea

a All participant characteristics listed were significantly associated with initial second-line antidiabetic medication class with a $P$-value of $<0.001$

b Poor health was defined according to the following criteria modified from the 2019 Endocrine Society Guidelines for diabetes management in older adults:

(1) end-stage conditions; (2) dementia; or (3) residence in a long-term nursing facility

c End-stage conditions included in the 2019 Endocrine Society's definition of poor health were: metastatic cancer, oxygen-dependent lung disease, chronic kidney disease stages IV or V, end-stage kidney disease, dialysis status, and advanced heart failure. Diagnostic codes used to define these conditions are listed in Table 2

d Diagnosis codes used to define dementia are listed in Table 2

e Residence in a long-term nursing facility was defined using Place of Service (POS) and CPT codes listed in Table 2

\section{Results}

After excluding those with type 1 diabetes $(n=28,014)$, secondary diabetes $(n=1,230)$, and patients who started more than $1 \mathrm{ADM}$ on the index date $(n=26,421)$, the final study cohort included 84,720 adults aged $\geq 65$ years with Medicare Advantage insurance plans. Among all participants with available values, the mean HbA1c prior to starting the index ADM was $7.95 \%( \pm 1.62)$. Over half of participants $(54 \%)$ received SFU as initial second-line ADM, 14\% received $\mathrm{BI}$, and $32 \%$ received another ADM. Table 4 reports statistically significant associations between all participant characteristics and index ADM class. Women accounted for a proportionately greater share of BI fills, and men were more likely to fill SFU. Participants aged 80 years and older were more likely to receive SFU and BI than other second-line ADM alternatives. A smaller proportion of SFU users had high DCSI scores and $\mathrm{HbA}_{1 \mathrm{c}}$ values $\geq 10 \%$, relative to other ADM. Conversely, $\mathrm{BI}$ fills were more common among those with a most recent $\mathrm{HbA}_{1 \mathrm{c}} \geq 10 \%$ or unavailable, and higher DCSI score. Most second-line ADM were prescribed by family practice or internal medicine clinicians, who demonstrated proportionally greater prescribing of SFU than other ADM alternatives. Endocrinologists accounted for a greater share of BI prescriptions than SFU. ADM fills were similar according to health plan, with proportionately more ADM alternatives filled in 2017 relative to SFU and BI.

Table 5 displays the bivariate association of the poor health status composite and its individual components with second-line ADM class. Overall, a small proportion of the older adult cohort met the definition of poor health $(6 \%)$, with a relatively equal share of participants meeting criteria for each of the following individual components that comprised poor health: end-stage chronic conditions (2\%), dementia (3\%), and residence in a nursing facility (2\%). Among those who filled BI as initial second-line ADM therapy, a greater share met the definition of poor health $(11 \%)$ and each of its individual components, namely end-stage chronic conditions (4\%), dementia (5\%), and residence in a nursing facility (6\%).

In multivariable models (Table 6), the odds of filling SFU or BI were higher for participants in poor health than those in good or intermediate health [OR 1.13 (95\% CI 1.05-1.21) and OR 2.34 (95\% CI 2.14-2.55), respectively]. Filling SFU as initial second-line ADM therapy was more common among men and in older age categories. By contrast, BI fills were more likely in women and less likely with increasing age categories, except for the oldest age category, which was not significant. SFU and BI were more commonly filled among older adults with poor glycemic control, which followed a 'dose-response' relationship with baseline HbA1c levels. This finding was most striking for $\mathrm{BI}$ among those with $\mathrm{HbA}_{1 \mathrm{c}} \geq 10.0 \%$ [OR 3.49 (95\% CI 3.12-3.90)]. Similarly, BI fills were also associated with diabetes severity as measured by the DCSI, with increasing odds for each successive score. SFU was more likely to be prescribed by family practice and internal medicine clinicians, whereas BI was more commonly prescribed by endocrinologists [OR 1.14 (95\% CI 1.03-1.25)]. Greater odds of filling BI was associated with health maintenance organization health plans. Generally, we observed decreasing odds of BI and SFU fills throughout the study period, which was significant for both ADM classes in 2017 compared with 2013.

In a sensitivity analysis removing race/ethnicity from the primary multivariable models, the primary findings did not substantively change. Separate multivariable models among only patients with available $\mathrm{HbA}_{1 \mathrm{c}}$ values, and adjusting for the most recent result, also yielded primary findings that were similar to those reported in Table 6 (data not shown). 
Table 6 Odds of Receiving SFU or BI as Initial Second-Line Antidiabetic Medication Class by Covariates

\begin{tabular}{|c|c|c|}
\hline Covariate & $\begin{array}{l}\text { SFU } \\
\text { OR (95\% Cl) }\end{array}$ & $\begin{array}{l}\text { BI } \\
\text { OR }(95 \% \mathrm{Cl})\end{array}$ \\
\hline \multicolumn{3}{|l|}{ Poor Health ${ }^{a}$} \\
\hline No & REF & REF \\
\hline Yes & $1.13(1.05-1.21)$ & $2.34(2.14-2.55)$ \\
\hline \multicolumn{3}{|l|}{ Sex } \\
\hline Male & REF & REF \\
\hline Female & $0.88(0.86-0.91)$ & $1.09(1.04-1.14)$ \\
\hline \multicolumn{3}{|l|}{ Age } \\
\hline $65-67$ years & REF & REF \\
\hline $68-70$ years & $0.98(0.93-1.02)$ & $0.83(0.77-0.89)$ \\
\hline 70-74 years & $1.07(1.03-1.12)$ & $0.85(0.80-0.90)$ \\
\hline 75-79 years & $1.18(1.12-1.24)$ & $0.93(0.86-0.99)$ \\
\hline$\geq 80$ years & $1.32(1.25-1.4)$ & $0.97(0.89-1.04)$ \\
\hline \multicolumn{3}{|l|}{ Hemoglobin $\mathrm{A}_{1 c}{ }^{\mathrm{b}}$} \\
\hline$<8 \%$ & REF & REF \\
\hline $8-10 \%$ & $1.18(1.12-1.24)$ & $1.19(1.08-1.31)$ \\
\hline$\geq 10 \%$ & $1.33(1.23-1.44)$ & $3.49(3.12-3.90)$ \\
\hline Unavailable & $1.53(1.47-1.60)$ & $3.35(3.12-3.60)$ \\
\hline \multicolumn{3}{|c|}{ Diabetes Complications Severity Index ${ }^{c}$} \\
\hline 0 & REF & REF \\
\hline 1 & $0.92(0.89-0.96)$ & $1.09(1.02-1.15)$ \\
\hline 2 & $0.95(0.91-1.00)$ & $1.12(1.04-1.20)$ \\
\hline$\geq 3$ & $0.92(0.87-0.96)$ & $1.25(1.16-1.34)$ \\
\hline \multicolumn{3}{|l|}{ Prescriber specialty } \\
\hline Family practice & REF & REF \\
\hline Endocrine & $0.50(0.47-0.54)$ & $1.14(1.03-1.25)$ \\
\hline General/Internal & $1.00(0.96-1.03)$ & $0.95(0.90-1.00)$ \\
\hline Nurse/PA & $0.85(0.80-0.90)$ & $1.10(1.02-1.20)$ \\
\hline Other/Missing & $0.99(0.95-1.04)$ & $1.21(1.13-1.30)$ \\
\hline \multicolumn{3}{|l|}{ Health Plan Type } \\
\hline Other & REF & REF \\
\hline Health maintenance organization & $1.03(0.99-1.07)$ & $1.25(1.18-1.31)$ \\
\hline Preferred provider organization & $1.06(1.00-1.11)$ & $0.88(0.81-0.96)$ \\
\hline \multicolumn{3}{|l|}{ Fill Year } \\
\hline 2013 & REF & REF \\
\hline 2014 & $0.86(0.81-0.92)$ & $1.08(0.98-1.18)$ \\
\hline 2015 & $0.80(0.75-0.85)$ & $0.90(0.82-0.99)$ \\
\hline 2016 & $0.90(0.85-0.95)$ & $1.28(1.18-1.39)$ \\
\hline 2017 & $0.69(0.65-0.73)$ & $0.91(0.84-0.99)$ \\
\hline
\end{tabular}

$B$ I basal insulin, SFU sulfonylurea

a Poor health was defined according to the following criteria modified from the 2019 Endocrine Society Guidelines for diabetes management in older adults: (1) end-stage conditions; (2) dementia; or (3) residence in a long-term nursing facility

b Laboratory values are not routinely available in health plan administrative data sources unless submitted by the laboratory vendor as part of their contract with the health payer. For these data, $35 \%$ of submitted laboratory claims nationally included a result. The Unavailable category includes patients without evidence of a test (no claim), as well as those with evidence of a test but no available result

c The Diabetes Complications Severity Index (DCSI) is a validated index composed of micro- and macrovascular complications of diabetes. In this analysis, we modified the DCSI by removing cardiovascular diagnoses that comprise the primary exposure variable. Scores were categorized as $0,1,2$, and $\geq 3$

\section{Discussion}

Practice guidelines for managing diabetes in older adults increasingly emphasize an individualized approach for determining appropriate glycemic targets and antidiabetic medication (ADM) regimens [3-7]. When making treatment decisions, clinical guidelines universally recommend assessing older patients' health status and considering potential associated risks, such as hypoglycemia [3-7]. Our study of 84,720 older adults found that the odds of filling SFU or BI was higher for participants in poor health than those in good or intermediate health, even after adjustment for the most recent $\mathrm{HbA}_{1 \mathrm{c}}$. Filling these same ADM classes was also more common among other subgroups of older adults. For example, SFU fills increased with advancing age; and BI fills increased with greater numbers of diabetes complications. Collectively, these findings demonstrate that SFU and BI remain widely used in older adults, including those who are particularly vulnerable for experiencing adverse events.

This analysis offers a unique perspective into real-world prescribing patterns for second-line ADM in older adults, with findings that are similar to a recent national analysis of electronic medical records [23]. Our use of administrative claims data enabled capture of medication fills, rather than prescription orders that are available from electronic health records but provide limited evidence about whether patients fill the medication. There is little research investigating the gap between existing practice patterns and current consensus guidelines on ADM use in older adults.

The poor health group in our analysis is of particular interest and has received little prior study, given that this population is almost universally excluded from clinical trials. Our findings suggest that SFU and BI are used frequently among older adults in poor health, which conflicts with multiple clinical guidelines recommending less frequent use of these medications among vulnerable older adults. One reason for this finding may be related to these medications' potent glucose-lowering effects [19]. However, short-term adverse events, including hypoglycemia, are frequently described among older adults in poor health, thereby compromising the future benefit of glycemic control in this group. Providers must consider the time horizon for treatment benefit in achieving glycemic targets and minimizing microvascular and macrovascular complications, such that therapy goals are attainable within patients' life expectancy, and treatment benefit outweighs risk of harm. Based on this assessment of risks and benefits, treatment with SFU and BI may still be appropriate among some older adults in poor health.

Previous studies have demonstrated that older adults with diabetes often receive more aggressive treatment than is warranted, reaching $\mathrm{HbA}_{1 \mathrm{c}}$ values below 
recommended targets [11, 13, 16, 17, 24]. Further, prior studies show low rates of medication de-intensification in older adults, highlighting a missed opportunity to reduce over-treatment [14, 15]. Several studies evaluate diabetes treatment according to older adults' health status. However, prior research has not described medication fills using the consensus framework that categorizes patients based on end-stage comorbid conditions, dementia, and/ or residence in long term care [11]. Because providers are accustomed to evaluating these comorbidities and characteristics [18, 25], the diabetes treatment framework originally developed by Blaum et al. [18] may prove useful in guiding development of individualized ADM regimens for older adults. Adherence to this framework, and resulting patient outcomes, should be studied empirically in future research that evaluates its implementation. Our findings demonstrate that, despite consensus guidelines from the AGS, ADA, and Endocrine Society recommending use of this framework, older adults in poor health were often treated with SFU and BI, highlighting the persistent gap that this recommendation aims to address.

This paradox is complex, and understanding it more completely requires further study. However, a few explanations and trends are evident. The enduring and frequent use of SFU therapy in older adults may be related to several factors, including its longstanding availability, prescriber familiarity, patient preference, significant glucose lowering effects, and low cost compared to many newer ADM alternatives [19]. The latter may be an especially important driver of SFU use, as these medications have the lowest cost of all ADM classes studied here [19]. Basal insulin is also an established therapy that may be an appropriate patient-centered treatment, especially for those with significantly elevated $\mathrm{HbA}_{1 \mathrm{c}}$ or contraindications to alternative therapy, such as end stage renal disease [19]. Prior research found that older adults with CKD, heart failure, and cardiovascular disease are less likely to fill SGLT-2 medications than those of younger age and those without such comorbid conditions, despite well documented clinical benefits [26]. It is not known whether this is related to concerns about side effects from newer medication classes among patients or providers, their higher cost, or providers' greater familiarity and experience with older ADM classes. Our study may have shown lower use of SGLT-2 and GLP-1 because the study period ended shortly after the publication of seminal trials demonstrating their cardiovascular and renal benefits. Using current data, future research must evaluate other reasons for infrequent use of newer ADM classes among older adults, and study ways to increase their uptake when clinically indicated.

Our study has notable limitations. Observational research is prone to confounding, which may limit causal inference from the findings. However, this issue is of greater potential concern when examining clinical outcomes rather than prescribing patterns examined in the current study. Our claims data source did not enable assessment of whether SFU and BI was clinically appropriate for some participants. Electronic health data have the potential to answer questions about the appropriateness of treatment decisions, given more available clinical information than claims. Hypoglycemia or falls were not examined as clinical outcomes here because these are underreported in claims data, and our group recently published an analysis focusing on glycemic outcomes of ADM using the same data source [10, 27]. Claims data do not routinely include laboratory results, although we were able to examine lab data for approximately one-third of participants. While we were unable to fully explore the relationship between $\mathrm{HbA}_{1 \mathrm{c}}$ level and choice of secondline $\mathrm{ADM}$, the primary findings were unchanged after adjusting for the most recent $\mathrm{HbA}_{1 \mathrm{c}}$ result among those with available $\mathrm{HbA}_{1 \mathrm{c}}$ data.

We analyzed data on participants' health plans, but did not have information about formularies or out-ofpocket costs that are known to influence ADM treatment decisions [28]. We used widely accepted definitions for dementia using diagnosis codes outlined by the Centers for Medicare and Medicaid Services [29]. These definitions may not have captured all participants with this exposure in our study, thereby underestimating the number of older adults in poor health. However, prior research on Medicare beneficiaries found that diagnosis codes are more sensitive for identifying older adults with dementia, which comprises the poor health group in the current study, than those with mild cognitive impairment [30]. Detailed data on functional impairment, which is another component of poor health status, is not available in administrative claims data. Prior studies have found that using diagnosis codes to define functional impairment exhibits poor sensitivity and specificity [31]. Clinical evaluation of functional status includes performance-based testing or survey data, which should be included in future research to capture patients with this exposure most completely.

\section{Conclusions}

In conclusion, our study documents high rates of SFU and BI use among all older adults and among particularly vulnerable subgroups, including those with advanced age, dementia, and high rates of diabetic complications. Our findings demonstrate a large opportunity to close the gap between real-world ADM prescribing patterns and current consensus guidelines that emphasize less frequent use of SFU and BI in older adults. This is especially true for those in poor health, 
for whom providers should consider alternative therapies and strategies for de-intensifying treatment. When initiating second-line ADM therapy, clinicians should follow recommended approaches for patient-centered care, using shared decision-making to discuss potential risks and benefits of different treatment options [32]. Future research should examine whether ADM prescribing patterns change in reaction to recent clinical guidelines. These studies should explore alternate data sources that allow for more complete capture of factors that define poor health in older adults. Future research on this topic will be informed by data from an ongoing clinical trial comparing the glycemic effects of most ADM classes studied here [33]. Population health efforts have the potential to improve clinical practice by examining data across health systems and using clinical decision support or other interventions that promote appropriate prescribing of SFU and BI. Emerging policies focused on reducing hypoglycemia also offer opportunities to align with diabetes treatment guidelines and incentivize care that limits adverse medication outcomes among older adults.

\section{Abbreviations}

ADM: Antidiabetic medications; SFU: Sulfonylureas; BI: Basal insulin; ADA: American Diabetes Association; AGS: American Geriatrics Society; DPP-4: dipeptidyl peptidase 4; GLP-1: glucagon-like peptide-1 angonists; SGLT-2: Sodium-glucose cotransporter 2 inhibiors; TZD: Thiazolidinediones; $\mathrm{HbA}_{1 \mathrm{c}}$ : Hemoglobin $A_{1 c} ;$ STROBE: Strengthening the Reporting of ObservationalStudies in Epidemiology ; DCSI: Diabetes Complications Severity Index.

\section{Acknowledgements}

Not applicable.

\section{Authors' contributions}

Study concept and design (KD, AW, RHK, DTL, RTA, MJO); acquisition of subjects and/or data (RHK, AC, MC, SAH, CA); analysis and interpretation of data (KD, AW, RHK, AC, MC, SAH, CA, DTL, RTA, MJO); and preparation of manuscript (KD, AW, RHK, AC, MC, SAH, CA, DTL, RTA, MJO). All authors have read and approved the manuscript.

\section{Funding}

This work was supported by a grant from United HealthCare Services, Inc. The study sponsor had no role in the design, methods, subject recruitment, data collections, analysis and preparation of paper.

\section{Availability of data and materials}

The datasets generated during and/or analyzed during the current study are available from the corresponding author on reasonable request.

\section{Declarations}

Ethics approval and consent to participate Institutional ethics review was conducted by the Northwestern University Institutional Review Board, which deemed this study to be non-human subjects research. Therefore participants' consent was not obtained. All methods were performed in accordance with the relevant guidelines and regulations.

\section{Consent for publication}

Not applicable.

\section{Competing interests}

AW has received research grant support from Eli Lilly and Company and Novo Nordisk A/S. The authors have no other potential conflicts of interest to report.

\section{Author details}

${ }^{1}$ Division of Endocrinology, Diabetes and Metabolism, Department of Medicine, University of Illinois at Chicago, 835 Wolcott St. Suite 625E M/C 640, IL 60612 Chicago, USA. ${ }^{2}$ Division of Endocrinology, Metabolism and Molecular Medicine, Department of Medicine, Northwestern University Feinberg School of Medicine, 300 E. Superior St. \#15-703, IL 60611 Chicago, USA. I Institute of Public Health and Medicine, Northwestern University Feinberg School of Medicine, 420 E. Superior St. 9th Floor, IL 60611 Chicago, USA. ${ }^{4}$ Division of General Internal Medicine and Geriatrics, Department of Medicine, Northwestern University Feinberg School of Medicine, 750 N. Lake Shore Dr., 10th floor, IL, Chicago, USA.

Received: 3 August 2021 Accepted: 24 January 2022

Published online: 03 February 2022

\section{References}

1. National Diabetes Statistics Report, Estimates of Diabetes and Its Burden in the United States. Centers for Disease Control and Prevention; U.S. Department of Health and Human Services, Atlanta, GA. 2020. https:// www.cdc.gov/diabetes/pdfs/data/statistics/national-diabetes-statisticsreport.pdf. Accessed Aug 82021.

2. Sinclair AJ, Abdelhafiz AH, Forbes A, Munshi M. Evidence-based diabetes care for older people with Type 2 diabetes: a critical review. Diabet Med. 2019;36:399-413.

3. LeRoith D, Biessels GJ, Braithwaite SS, Casanueva FF, Draznin B, Halter JB et al. Treatment of Diabetes in Older Adults: An Endocrine Society* Clinical Practice Guideline. J Clin Endocrinol Metab. 2019;104(5):1520-74.

4. Kirkman MS, Briscoe VJ, Clark N, Florez H, Haas LB, Halter JB et al. Diabetes in older adults: a consensus report. J Am Geriatr Soc. 2012;60(12):2342-56.

5. Sinclair AJ, Abdelhafiz A, Dunning T, Izquierdo M, Rodriguez Manas L, Bourdel-Marchasson I et al. An International Position Statement on the Management of Frailty in Diabetes Mellitus: Summary of Recommendations 2017. J Frailty Aging. 2018;7(1):10-20.

6. Older Adults: Standards of Medical Care in Diabetes-2020. Diabetes Care. 2020;43(Suppl 1):S152-s62.

7. Conlin PR, Colburn J, Aron D, Pries RM, Tschanz MP, Pogach L. Synopsis of the 2017 U.S. Department of Veterans Affairs/U.S. Department of Defense Clinical Practice Guideline: Management of Type 2 Diabetes Mellitus. Ann Intern Med. 2017;167(9):655-63.

8. Huang ES, Laiteerapong N, Liu JY, John PM, Moffet HH, Karter AJ. Rates of complications and mortality in older patients with diabetes mellitus: the diabetes and aging study. JAMA Intern Med. 2014;174(2):251-8.

9. Lee AK, Lee CJ, Huang ES, Sharrett AR, Coresh J, Selvin E. Risk Factors for Severe Hypoglycemia in Black and White Adults With Diabetes: The Atherosclerosis Risk in Communities (ARIC) Study. Diabetes Care. 2017:40(12):1661-7.

10. Wallia A, O'Brien MJ, Liss DT, Kang RH, Cooper AJ, Gilmer A et al. Glycemic Outcomes of Second Line Diabetes Drug Choice in a Real World Population. Mayo Clin Proc Inn Qual Out. 2021;5(3):675-81.

11. Lipska KJ, Ross JS, Miao Y, Shah ND, Lee SJ, Steinman MA. Potential overtreatment of diabetes mellitus in older adults with tight glycemic control. JAMA Intern Med. 2015;175(3):356-62.

12. Arnold SV, Lipska KJ, Wang J, Seman L, Mehta SN, Kosiborod M. Use of Intensive Glycemic Management in Older Adults with Diabetes Mellitus. J Am Geriatr Soc. 2018;66(6):1190-4.

13. Hambling CE, Seidu SI, Davies MJ, Khunti K. Older people with Type 2 diabetes, including those with chronic kidney disease or dementia, are commonly overtreated with sulfonylurea or insulin therapies. Diabet Med. 2017;34(9):1219-27.

14. Maciejewski ML, Mi X, Sussman J, Greiner M, Curtis LH, Ng J et al. Overtreatment and Deintensification of Diabetic Therapy among Medicare Beneficiaries. J Gen Intern Med. 2018;33(1):34-41. 
15. Sussman JB, Kerr EA, Saini SD, Holleman RG, Klamerus ML, Min LC et al. Rates of Deintensification of Blood Pressure and Glycemic Medication Treatment Based on Levels of Control and Life Expectancy in Older Patients With Diabetes Mellitus. JAMA Intern Med. 2015;175(12):1942-9.

16. Thorpe CT, Gellad WF, Good CB, Zhang S, Zhao X, Mor M et al. Tight glycemic control and use of hypoglycemic medications in older veterans with type 2 diabetes and comorbid dementia. Diabetes Care. 2015;38(4):588-95.

17. Tseng CL, Soroka O, Maney M, Aron DC, Pogach LM. Assessing potential glycemic overtreatment in persons at hypoglycemic risk. JAMA Intern Med. 2014;174(2):259-68.

18. Blaum C, Cigolle CT, Boyd C, Wolff JL, Tian Z, Langa KM et al. Clinical complexity in middle-aged and older adults with diabetes: the Health and Retirement Study. Med Care. 2010;48(4):327-34.

19. Ackermann RT, Wallia A, O'Brien MJ, Kang R, Cooper A, Moran MR et al. Correlates of second-line type 2 diabetes medication selection in the USA. BMJ Open Diabetes Research \& Care. 2017;5(1):e000421.

20. von Elm E, Altman DG, Egger M, Pocock SJ, Gøtzsche PC, Vandenbroucke JP. The Strengthening the Reporting of Observational Studies in Epidemiology (STROBE) Statement: guidelines for reporting observational studies. Int J Surg. 2014;12(12):1495-9.

21. Lund JL, Richardson DB, Sturmer T. The active comparator, new user study design in pharmacoepidemiology: historical foundations and contemporary application. Curr Epidemiol Rep. 2015;2(4):221-8.

22. Young BA, Lin E, Von Korff M, Simon G, Ciechanowski P, Ludman EJ et al. Diabetes complications severity index and risk of mortality, hospitalization, and healthcare utilization. Am J Manag Care. 2008;14(1):15-23.

23. Montvida O, Shaw J, Atherton JJ, Stringer F, Paul SK. Long-term Trends in Antidiabetes Drug Usage in the U.S.: Real-world Evidence in Patients Newly Diagnosed With Type 2 Diabetes. Diabetes Care. 2018;41(1):69-78.

24. McCoy RG, Lipska KJ, Van Houten HK, Shah ND. Paradox of glycemic management: multimorbidity, glycemic control, and high-risk medication use among adults with diabetes. BMJ Open Diabetes Res Care. 2020;8(1):e001007. https://doi.org/10.1136/bmjdrc-2019-001007.

25. Cigolle CT, Kabeto MU, Lee PG, Blaum CS. Clinical complexity and mortality in middle-aged and older adults with diabetes. J Gerontol A Biol Sci Med Sci. 2012;67(12):1313-20

26. McCoy RG, Dykhoff HJ, Sangaralingham L, Ross JS, Karaca-Mandic P, Montori VM et al. Adoption of New Glucose-Lowering Medications in the U.S.-The Case of SGLT2 Inhibitors: Nationwide Cohort Study. Diabetes Technol Ther. 2019;21(12):702-12.

27. Hoffman GJ, Ha J, Alexander NB, Langa KM, Tinetti M, Min LC. Underreporting of Fall Injuries of Older Adults: Implications for Wellness Visit Fall Risk Screening. J Am Geriatr Soc. 2018;66(6):1195-200.

28. Karter AJ, Parker MM, Solomon MD, Lyles CR, Adams AS, Moffet $\mathrm{HH}$ et al. Effect of Out-of-Pocket Cost on Medication Initiation, Adherence, and Persistence among Patients with Type 2 Diabetes: The Diabetes Study of Northern California (DISTANCE). Health Serv Res. 2018;53(2):1227-47.

29. Centers for Medicare \& Medicaid Services. Chronic Conditions Data Warehouse, Condition Categories Alzheimer's Disease and Related Disorders or Senile Dementia. 2021. Accessed May 212021

30. Taylor DH, Jr., Fillenbaum GG, Ezell ME. The accuracy of medicare claims data in identifying Alzheimer's disease. J Clin Epidemiol. 2002;55(9):929-37.

31. Ben-Shalom Y, Stapleton DC. Predicting Disability among CommunityDwelling Medicare Beneficiaries Using Claims-Based Indicators. Health Serv Res. 2016;51(1):262-81.

32. Tamhane S, Rodriguez-Gutierrez R, Hargraves I, Montori V. Shared Decision-Making in Diabetes Care. Curr Diab Rep. 2015;15(12):1-10

33. Wexler, DJ, Krause-Steinrauf H, Crandall JP, Florez HJ, Hox SH Kuhn A, et al. Baseline Characteristics of Randomized Participants in the Glycemia Reduction Approaches in Diabetes: A Comparative Effectiveness Study (GRADE). Diab Care. 2019;42:2098-2107.

\section{Publisher's Note}

Springer Nature remains neutral with regard to jurisdictional claims in published maps and institutional affiliations.
Ready to submit your research? Choose BMC and benefit from:

- fast, convenient online submission

- thorough peer review by experienced researchers in your field

- rapid publication on acceptance

- support for research data, including large and complex data types

- gold Open Access which fosters wider collaboration and increased citations

- maximum visibility for your research: over $100 \mathrm{M}$ website views per year

At BMC, research is always in progress.

Learn more biomedcentral.com/submissions 